\section{TO MET OR NOT TO MET: THAT IS THE QUESTION!}

M Sulistio, ${ }^{1}$ A Vo, ${ }^{1}$ M Franco ${ }^{1}{ }^{1}$ McCulloch House Supportive \& Palliative Care Unit, Southern Health, Melbourne; ${ }^{2}$ Palliative Care Consultancy, Eastern Health, Melbourne, ${ }^{3}$ Monash University, Melbourne

10.1136/bmjspcare-2013-000491.37

Background The Medical Emergency Team (MET) aim to identify clinically deteriorating patients to provide early intervention and prevent negative outcomes. However, for a proportion of these patients (ie, patients who are expectedly dying) continued intervention is 'medically futile' and unwanted by the patient and/or next-of-kin. Although diagnosing dying can be complex and challenging, it is an important step to establish appropriate goal of care, avoid futile medical intervention and allow a 'good death'.

Aim

- To determine the incidence of MET or Code Blues on patients who were expectedly dying.

- To identify patient's, clinician's or institution's variables that result in the occurrence of such incidents

Methods Retrospective 3 months audit of MET and code blue occurring on all Southern Health inpatients with onsite access to ICU support (Monash Medical
Centre Clayton, Jessie McPherson Private Hospital, and Dandenong Hospital). Medical records were reviewed to collect patient demographics, admission and incident details, existing life limiting diagnosis, resuscitation and limitation of medical therapy status, clinical observations that may suggest terminal phase ${ }^{\$}$, and documentation of communication with patient's next-of-kin.

Results A total of 456 incidents on 351 adult patients are analysed.

Conclusion A significant proportion of MET (10\%) in a major hospital network may have been avoided if dying patients were better identified and the documentation of the goals of care was clearer. More such incidents occurred out of business hours than within hours. These results should encourage prospective study into what further measures could reduce this proportion of incidents.

\begin{tabular}{|c|c|c|c|c|}
\hline & Expectedly dying $\$$ & Not expected to die & GOC $^{\#}$ changed & $\mathrm{GOC}^{\#}$ unchanged \\
\hline $\begin{array}{l}\text { Incidents (patients) } \\
\mathrm{AH} \wedge(\%) \\
\text { - Multiple/admit (\%) }\end{array}$ & $\begin{array}{l}47(38) \\
31(66 \%) \\
22(47 \%)\end{array}$ & $\begin{array}{l}409(318) \\
241(59 \%) \\
161(39 \%)\end{array}$ & $\begin{array}{l}104(95) \\
60(58 \%) \\
42(40 \%)\end{array}$ & $\begin{array}{l}352(282) \\
212(60 \%) \\
141(40 \%)\end{array}$ \\
\hline $\begin{array}{l}\text { Demographics } \\
\text { Age (Range) } \\
\text { Male\% } \\
\text { - From HLC\%* }\end{array}$ & $\begin{array}{l}72.05(28-96) \\
50 \% \\
18 \%\end{array}$ & $\begin{array}{l}66.7(18-95) \\
56 \% \\
5.3 \%\end{array}$ & $\begin{array}{l}76.9(31-96) \\
61 \% \\
19 \%\end{array}$ & $\begin{array}{l}64.7(18-96) \\
51 \% \\
4 \%\end{array}$ \\
\hline $\begin{array}{l}\text { Admission (\%) } \\
\text { Medical } \\
-\mathrm{AH}^{\wedge}\end{array}$ & $\begin{array}{l}70 \% \\
61 \%\end{array}$ & $\begin{array}{l}66 \% \\
54 \%\end{array}$ & $\begin{array}{l}77 \% \\
55 \%\end{array}$ & $\begin{array}{l}63 \% \\
54 \%\end{array}$ \\
\hline $\begin{array}{l}\text { Life-limiting illness (\%) } \\
\text { Malignant } \\
\text { - Non-malignant }\end{array}$ & $\begin{array}{l}21 \% \\
47 \%\end{array}$ & $\begin{array}{l}13 \% \\
31 \%\end{array}$ & $\begin{array}{l}25 \% \\
49 \%\end{array}$ & $\begin{array}{l}10.8 \% \\
33 \%\end{array}$ \\
\hline $\begin{array}{l}\text { Outcome }(\%) \\
\text { Death } \\
\text { Altered SOC }{ }^{\&}\end{array}$ & $\begin{array}{l}74 \% \\
2.6 \% \\
\end{array}$ & $\begin{array}{l}20 \% \\
25.5 \% \\
\end{array}$ & $\begin{array}{l}53 \% \\
25 \% \\
\end{array}$ & $\begin{array}{l}19 \% \\
13.8 \% \\
\end{array}$ \\
\hline
\end{tabular}

\$Patients with 2 out of 4 clinical observations prior to incident (taking sips of fluid only, unable to swallow medications, bed-bound, semi-comatose) ${ }^{\#}$ Goal of care $\wedge$ After hours (weekend/public holiday and weekday outside of $0800-1700 \mathrm{~h}$ ) ${ }^{*}$ High level care \& Site of Care (rehabilitation, residential care facility or inter-hospital transfer). 


\section{Corrections}

Sulistio M, Vo A, Franco M et al. To MET or not to MET: That is the question! BMJ Support Palliat Care 2013;3:239. Leeroy William has been added as an author.

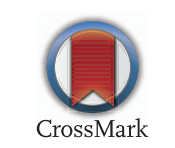

BMJ Supportive \& Palliative Care 2015;5:206. doi:10.1136/bmjspcare-2013-000491.37corr1 